\title{
Invasive mucinous carcinoma of the breast
}

\author{
Kelli Y. Ha, MD, Patricia DeLeon, D0, and William DeLeon, MD
}

\begin{abstract}
Mucinous carcinoma of the breast is one of the rarer forms of intramammary cancer, often presenting as a lobulated, fairly well circumscribed mass on mammography, sonography, and gadolinium-enhanced magnetic resonance imaging. It accounts for $1 \%$ to $7 \%$ of all breast cancers and generally carries a better prognosis than other types of malignant breast cancers. Metastatic disease occurs at a lower frequency than in other types of invasive carcinoma. We present an atypical case of mucinous carcinoma in a woman who presented with a palpable intramammary lymph node metastasis from an unknown breast primary. Subsequent magnetic resonance imaging and percutaneous biopsy demonstrated histologic findings consistent with a mixed mucinous neoplasm with a micropapillary pattern.
\end{abstract}

nvasive mucinous carcinoma of the breast is one of the rarer breast neoplasms and is typically associated with a better prognosis than other types of breast cancer. It often presents as a lobulated and/or well-circumscribed mass on mammography, sonography, and magnetic resonance imaging (MRI). We present the case of a patient with a more aggressive type of mucinous carcinoma: a mixed neoplasm with micropapillary features.

\section{CASE REPORT}

A 52-year-old woman presented to her primary care physician complaining of a new lump in the upper outer quadrant of her left breast. A mammogram demonstrated a $7 \mathrm{~mm}$ ovoid mass at the 2:00 position of the left breast (Figure 1). Targeted sonographic evaluation demonstrated a well-circumscribed, oval-shaped mass of mixed echogenicity at the 2:00 position, approximately $10 \mathrm{~cm}$ from the nipple (Figure 2). Ultrasoundguided percutaneous biopsy results were consistent with metastatic involvement of an intramammary lymph node from a mammographically occult breast carcinoma.

Gadolinium-enhanced breast MRI demonstrated a $3.9 \times$ $5.2 \times 1.7 \mathrm{~cm}$ area of suspicious nonmasslike enhancement within the lower inner and lower outer quadrants of the left breast. A $1.2 \mathrm{~cm}$ focus of masslike enhancement was also visualized at the 7:00 position of the left breast (Figure 3). These two foci of abnormal enhancement were subsequently biopsied; both sites were consistent with an infiltrating ductal carcinoma (grade I/II) with mucinous and micropapil-

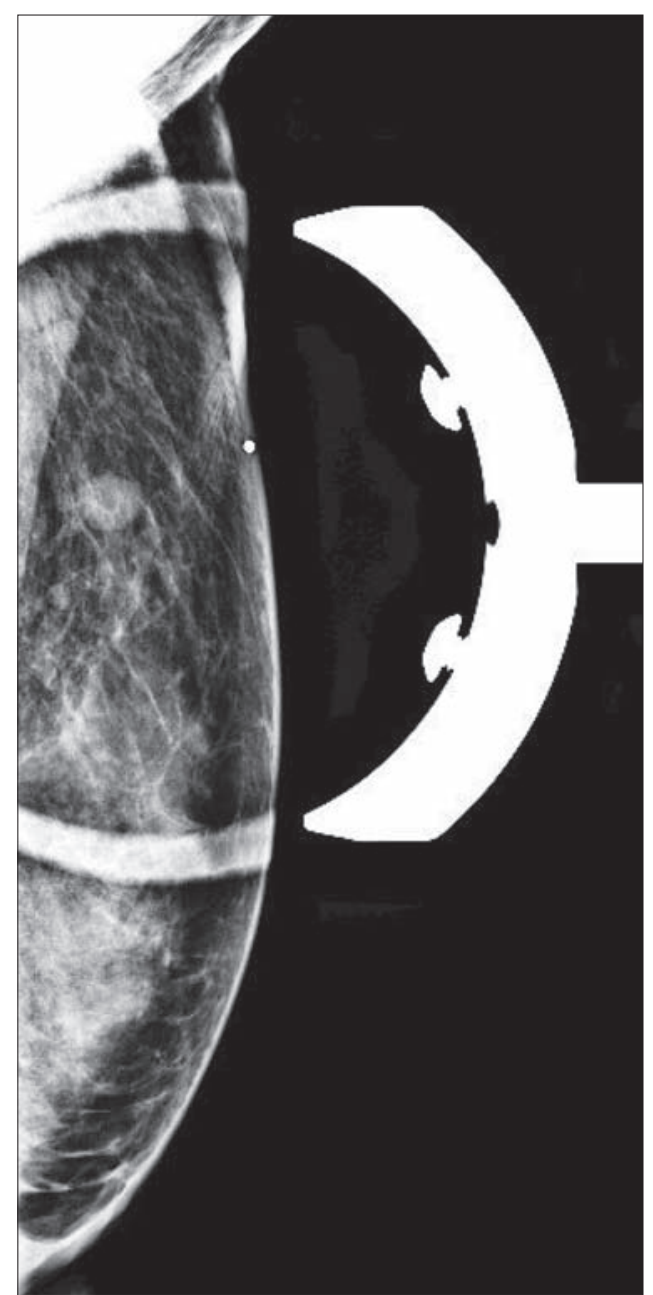

Figure 1. Spot mammographic view of the left breast demonstrates a $7 \mathrm{~mm}$ ovoid mass at the palpable area of concern as designated by a radiopaque marker.

lary patterns. The areas of tumor were found in association with extensive tumor emboli within dermal lymphatics, suggestive of widespread neoplastic involvement of the left breast. The lesion was estrogen and progesterone receptor

From the Department of Radiology, Baylor University Medical Center at Dallas. Corresponding author: Kelli Y. Ha, MD, Department of Radiology, Baylor University Medical Center at Dallas, 3500 Gaston Avenue, Dallas, TX 75246 (e-mail: yeek816@gmail.com). 


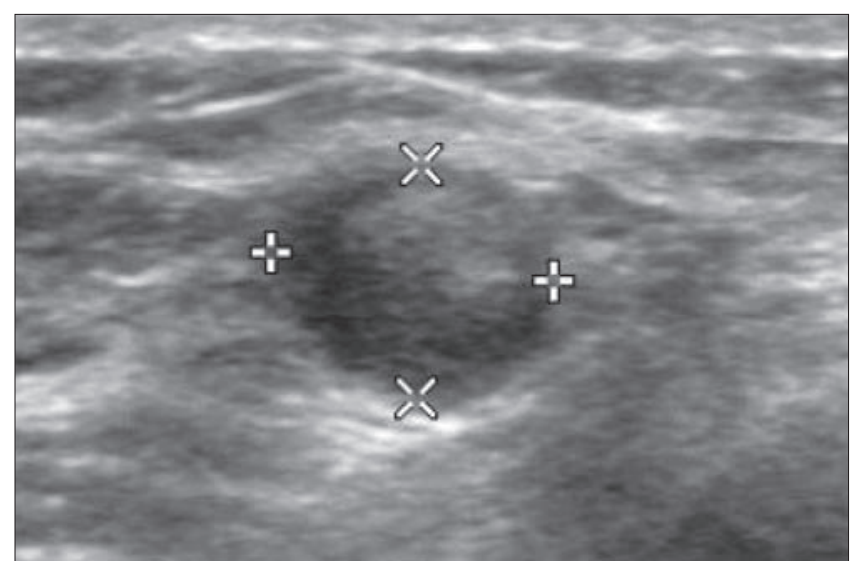

Figure 2. Sonographic image of the left breast demonstrates a $7 \times 6 \mathrm{~mm}$ mixed echogenicity ovoid mass at the 2:00 position, approximately $10 \mathrm{~cm}$ from the nipple. Histologic sampling yielded findings consistent with a metastatic lymph node from an occult breast primary.

(ER/PR) negative and HER-2 positive (3+ staining). Bone scintigraphy and gadolinium-enhanced brain MRI were negative for extramammary spread of disease. The patient is presently exploring various treatment options with her medical oncologist.

\section{DISCUSSION}

Mucinous (colloid) carcinoma of the breast is one of the rarer breast neoplasms, accounting for $1 \%$ to $7 \%$ of all invasive breast carcinomas. A prevalence as high as $7 \%$ is found in women over the age of 75 years, while a prevalence of $1 \%$ is found in those younger than 35 . A mucin component $>33 \%$ to $50 \%$ defines this type of tumor (1). From a histologic perspective, mucinous carcinomas may be divided into pure mucinous and mixed mucinous entities, depending upon the percentage of mucin within the neoplasm. Pure mucinous carcinomas (mucin
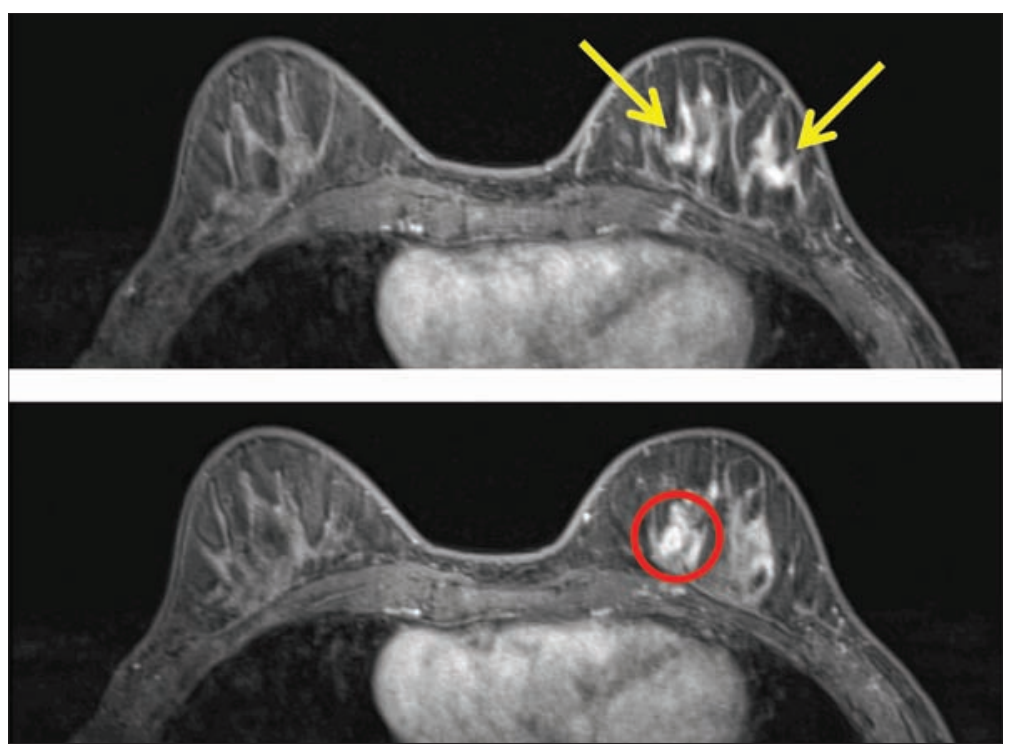

Figure 3. Two gadolinium-enhanced MR images of the left breast demonstrate multiple areas of nonmasslike enhancement (yellow arrows) within the lower inner and lower outer quadrants of the left breast. An area of masslike enhancement (red circle) is also identified at the 7:00 position.
Table. Characteristics of mucinous breast neoplasms visible on mammography over a 3-year period at Baylor University Medical Center at Dallas*

\begin{tabular}{ccccc}
\hline Patient & Age (years) & Breast & Size (cm) & Palpable \\
\hline 1 & 61 & Left & 1.0 & 0 \\
2 & 77 & Left & 0.9 & 0 \\
3 & 80 & Right & 1.2 & 0 \\
4 & Left & $1.8 / 0.8$ & + \\
5 & 61 & Left & 1.2 & 0 \\
6 & 80 & Right & 1.3 & 0 \\
7 & 74 & Right & 0.9 & 0 \\
8 & 75 & Right & 2.1 & 0 \\
9 & 51 & Right & 4.5 & 0 \\
10 & 55 & Right & 0.8 & 0 \\
11 & 64 & Right & - & 0 \\
12 & 49 & Left & 1.6 & + \\
13 & 65 & Right & 0.6 & 0 \\
14 & 60 & Left & 2.5 & 0 \\
\hline
\end{tabular}

*Data cover the period from April 1, 2008, to March 31, 2011, and exclude the recent patient discussed in the case report. Mucinous carcinomas comprised 14 of 560 breast neoplasms, constituting a $2.5 \%$ prevalence rate over the 3 -year period. Three of the 14 mucinous neoplasms (21\%) were of a mixed mucinous type.

- indicates no information available.

component $>90 \%$ ) are associated with a better prognosis, a longer disease-free interval, and a lower incidence of axillary node metastasis $(2,3)$. The frequency of mucinous intramammary neoplasms at Baylor University Medical Center at Dallas is summarized in the Table, with a reported incidence over the 3 -year period of approximately $2.5 \%$.

When palpable, these cancers often present as soft masses due to their semisolid mucin constituents. Most mucinous carcinomas are readily detected on mammography. They appear as low-density, well-defined or microlobulated oval masses and generally belong to the category of "well-circumscribed" breast carcinomas (4). Microlobulated margins have been associated with higher mucin content, while irregular or spiculated margins correspond to lower percentages of mucin and infiltrating margins histologically. The irregular and infiltrating margins seen on mammography and histology have been attributed to greater degrees of fibrosis associated with the nonmucinous components (5). Albeit rare, calcifications seen in conjunction with mucinous tumors frequently correspond to the invasive ductal component of the cancer in a mixed mucinous tumor (6).

Sonographically, mucinous carcinomas typically present as complex masses of mixed echogenicity with solid and cystic-appearing components. However, up to $20 \%$ of these lesions may present as homogenous masses on ultrasound. They are isoechoic or hypoechoic to subcutaneous fat, with posterior acoustic 
enhancement a common finding. Microlobulated contour is often more readily demonstrated on sonography rather than mammography $(3,4)$.

Mucinous carcinomas of the mammary gland frequently present as lobulated tumors on MRI, with dynamic time-signal intensity curves that exhibit a gradual contrast enhancement or plateau-type pattern after the initial upstroke. They classically demonstrate high signal intensity on T2-weighted images due to the intrinsic mucin component. Signal intensity on T1-weighted images varies from low to high, a feature that is largely dependent on the protein composition of the tumor $(4,7)$.

The overall survival of patients with mucinous carcinoma differs little from age-matched individuals within the general population $(8,9)$. More current studies have shown that a subset of patients diagnosed with mucinous carcinoma do not demonstrate such favorable outcomes. In particular, Barbashina et al (10) and Shet et al (11) revealed that specific subtypes of pure mucinous carcinoma - those with a micropapillary patterndemonstrate significantly worse prognoses. A micropapillary pattern consists of an "inside-out" arrangement of tumor cells surrounded by clear spaces with a high propensity for angioinvasion. In the study of Barbashina et al (10), more than half of patients with this type of pattern were found to have lymphovascular invasion, and synchronous axillary lymph node metastases were detected in approximately one third. Twenty percent of patients also demonstrated HER-2 and P53 positivity, factors that are generally associated with a worse prognosis (10). High nuclear grade was noted in association with this type of micropapillary pattern in a separate study by Ranade et al (12).

Recent studies $(11,13)$ have also revealed that a subset of patients diagnosed with mucinous carcinoma present with mammographically occult neoplasms and/or multifocal/multicentric disease. Additionally, some of these patients demonstrate metastases at presentation, as well as tumors that are ER/ PR negative and HER-2 positive. Traditionally, pure mucinous carcinomas exhibit a rate of metastasis of $<15 \%$ (8). In addition, these neoplasms classically demonstrate ER/PR positivity and HER-2 negativity, characteristics that often confer a better prognosis. Although research is limited at this point, a heightened recognition of the biologic heterogeneity of this tumor may lead to improved use of radiologic modalities, more individualized treatment, and improved outcomes in a certain subset of patients.

1. Bae SY, Choi MY, Cho DH, Lee JE, Nam SJ, Yang JH. Mucinous carcinoma of the breast in comparison with invasive ductal carcinoma: clinicopathologic characteristics and prognosis. J Breast Cancer 2011;14(4):308-313.

2. Cardenosa G, Doudna C, Eklund GW. Mucinous (colloid) breast cancer: clinical and mammographic findings in 10 patients. AJR Am J Roentgenol 1994;162(5):1077-1079.

3. Lam WW, Chu WC, Tse GM, Ma TK. Sonographic appearance of mucinous carcinoma of the breast. AJR Am J Roentgenol 2004;182(4):1069-1074.

4. Harvey JA. Unusual breast cancers: useful clues to expanding the differential diagnosis. Radiology 2007;242(3):683-694.

5. Wilson TE, Helvie MA, Oberman HA, Joynt LK. Pure and mixed mucinous carcinoma of the breast: pathologic basis for differences in mammographic appearance. AJR Am J Roentgenol 1995;165(2):285-289.

6. Conant EF, Dillon RL, Palazzo J, Ehrlich SM, Feig SA. Imaging findings in mucin-containing carcinomas of the breast: correlation with pathologic features. AJR Am J Roentgenol 1994;163(4):821-824.

7. Kawashima M, Tamaki Y, Nonaka T, Higuchi K, Kimura M, Koida T, Yanagita Y, Sugihara S. MR imaging of mucinous carcinoma of the breast. AJR Am J Roentgenol 2002;179(1):179-183.

8. Diab SG, Clark GM, Osborne CK, Libby A, Allred DC, Elledge RM. Tumor characteristics and clinical outcome of tubular and mucinous breast carcinomas. J Clin Oncol 1999;17(5):1442-1448.

9. Yerushalmi R, Hayes MM, Gelmon KA. Breast carcinoma-rare types: review of the literature. Ann Oncol 2009;20(11):1763-1770.

10. Barbashina V, Corben AD, Akram M, Vallejo C, Tan LK. Mucinous micropapillary carcinoma of the breast: an aggressive counterpart to conventional pure mucinous tumors. Hum Pathol 2013 Mar 18 [Epub ahead of print].

11. Shet T, Chinoy R. Presence of a micropapillary pattern in mucinous carcinomas of the breast and its impact on the clinical behavior. Breast $J$ 2008;14(5):412-420.

12. Ranade A, Batra R, Sandhu G, Chitale RA, Balderacchi J. Clinicopathological evaluation of 100 cases of mucinous carcinoma of breast with emphasis on axillary staging and special reference to a micropapillary pattern. J Clin Pathol 2010;63(12):1043-1047.

13. Perkins G, Babiera G, Bedrosian I, Gonzalez-Angulo A, Whitman G, Yang W, Strom E, Woodward W, Tereffe W, Yu T, Oh J, Buchholz T, Middleton L. Mucinous breast carcinoma: occult multifocality/multicentricity in a favorable disease. Cancer Res 2009;69(24 Suppl):4117. 